Check for updates

Cite this: RSC Adv., 2017, 7, 22461

\title{
Reconfigurable photonic crystals with optical bistability enabled by "cold" programming and thermo-recoverable shape memory polymers $\uparrow$
}

\begin{abstract}
Wenbin Niu, Lingcheng Qu, Rongwen Lyv and Shufen Zhang (DD *
Recently, reconfigurable photonic crystals that can reversibly change and recover their optical properties (e.g. photonic stopbands) in response to external stimuli have aroused much attention. However, developing a photonic crystal with a dynamically tunable nanostructure that can memorize and maintain different nanostructures and related optical properties is still a challenge. Based on capillary pressureinduced "cold" programming and heat-triggered recoverable shape memory polymers, reconfigurable 3D photonic crystal membranes with optical bistability, which show two reversibly tunable stable equilibrium states, are reported in this work. During shape memory programming and recovery processes, capillary pressure created by water evaporation induces "cold" programming of photonic crystal membranes with a disordered temporary state without diffractive peaks at room temperature. The permanent ordered photonic crystal nanostructures with apparent characteristic diffraction peaks can be recovered by heating. The reversible transition of photonic crystals between an ordered permanent state and a disordered temporary state during the cyclical programming and recovery results in a tremendous change in appearance and a large and stable shift of diffraction peaks different from conventionally tunable photonic crystals. This disorder-to-order transition and the tremendous shift of photonic bandgaps enable the photonic crystals to serve as a sensitive optical tool to the probe shape memory effect at the nanoscale. Importantly, a large difference in optical transmittances is shown as no stopband exists in the disordered temporary state, which provides a unique alternative in developing photonic crystal photoswitches with higher contrast and optical switching effect, as well as rewritable photonic devices and displays etc.
\end{abstract}

Received 26th December 2016

Accepted 11th April 2017

DOI: $10.1039 / c 6 r a 28682 h$

rsc.li/rsc-advances lattice constants of $\mathrm{Fe}_{3} \mathrm{O}_{4} @ \mathrm{SiO}_{2}$ and $\mathrm{ZnS} @ \mathrm{SiO}_{2}$ photonic crystal assemblies, ${ }^{27-29}$ thus achieving tunable photonic stopbands for full-color displays. For commonly adjustable photonic crystals, however, the temporarily configured nanostructures and optical states cannot be memorized, which will easily recover to their pristine states once the external stimuli are ceased. , $^{\mathbf{6 , 2 3 , 3 0}}$

By contrast, optically bistable photonic crystals show two reversibly stable equilibrium states, on which various optically stable microstructures could be patterned, erased and rewritten. This capability would greatly simplify the fabrication process of microoptical devices and facilitate the development of devices that require reconfigured optically stable states (such as micropatternings, optical circuits). ${ }^{31-34}$ Shape memory polymers, which can memorize temporary shape and revert to their permanent shape upon exposure to an external stimulus, ${ }^{35-37}$ are thought as ideal materials to construct reconfigurable photonic crystals with optical bistability. In a typical thermoresponsive shape memory cycle, a temporary shape is first "programmed" from a permanent shape at a temperature higher than the transition temperature (such as $T_{\mathrm{g}}$ ). The temporary shape is then fixed by cooling the sample below $T_{\mathrm{g}}$, and remains stable indefinitely. The permanent shape can be
State Key Laboratory of Fine Chemicals, Dalian University of Technology, West Campus, 2 Linggong Rd., Dalian 116024, China. E-mail: zhangshf@dlut.edu.cn

$\dagger$ Electronic supplementary information (ESI) available. See DOI: $10.1039 / \mathrm{c} 6 \mathrm{ra} 28682 \mathrm{~h}$ 
recovered by heating above $T_{\mathrm{g}}$ via entropy elasticity mechanism. ${ }^{35,38}$ Based on thermoresponsive shape memory polymers, shape-memorizing microprism arrays and 2D grating patterns have been reported recently, by thermal embossing nanoimprint lithography at $180{ }^{\circ} \mathrm{C} .{ }^{39}$ Similarly, 2D photonic nanostructure with hexagonal nanobowls array on the surface of a thermal shape-memory elastomer was also fabricated. ${ }^{\mathbf{4 0}}$ However, such photonic nanostructures showed low reflective amplitudes of optical stopbands due to $2 \mathrm{D}$ nature of those surface arrays. To improve optically reflective performance, Jiang and co-workers introduced 3D inverse opal photonic crystals by using polyurethane thermoresponsive copolymer, ${ }^{\mathbf{4 1}}$ as $3 \mathrm{D}$ ordered nanostructures with high crystalline would exhibit much stronger light diffraction. ${ }^{3,8,13}$ Unfortunately, heating is still utilized to realize programming for these devices. By contrast, "cold" programming at room temperature will greatly improve the processability of shape-memorizing photonic devices. Although great efforts have been made, "cold" programming reconfigurable 3D photonic crystals with optical bistability are rarely reported and the related work is still in its infancy. ${ }^{41-43}$

Herein, in this study, we present the reconfigurable 3D photonic crystal membranes with optically bistable states, which were enabled by "cold" programming at room temperature and heating-recoverable shape memory polymers. Capillary pressure produced by water evaporation induced "cold" programming of photonic crystal membranes at room temperature. This capillary pressure together with heating-recovery resulted in reversible transition of photonic crystal between a disordered temporary state and an ordered permanent state, thus creating a tremendous change in optically reflective properties, as well as a higher transmittance contrast than conventional photonic crystals, which implies great promise for applications in displays, photoswitches etc.

\section{Results and discussion}

Fig. 1 illustrates the configuration and transition of a 3D ordered inverse opal photonic crystal membrane with optically bistable states, via a "cold" programming and heat-recovery procedure. These photonic crystal membranes were prepared by using silica opals as templates, which were self-assembled by vertical deposition method using high-charged silica nanospheres with diameter ranging from 230 to $330 \mathrm{~nm} .^{\mathbf{9 , 4 4 , 4 5}}$ The thickness of the opal template was controlled to $3 \mu \mathrm{m}$ or so by

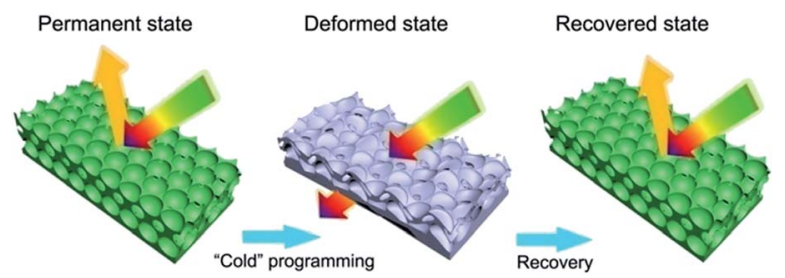

Fig. 1 Schematic illustration of "cold" programming and recovery procedure of 3D ordered inverse opal photonic crystal membrane with optically bistable states. adjusting the concentration of ethanol suspension. Then, the templates were infiltrated with viscous mixtures of commercialized ethoxylated (15) trimethylolpropane triacrylate (EO15TMPTA, refractive index 1.470) and polyethylene glycol (600) diacrylate (PEG600DA, refractive index 1.468) (Fig. S1†) with various weight ratios from $1: 2$ to $1: 6$. As the optimally reconfigurable and optically bistable behaviors were shown in EO15TMPTA-co-PEG600DA copolymer with 1:4 ratio, this ingredient was used in this work if not specifically stated otherwise. After UV polymerization, free-standing macroporous inverse opal photonic crystal membranes of copolymer were obtained by soaking these samples in hydrofluoric acid aqueous solution to selectively remove silica templates. The resulting inverse opal photonic crystal membranes consists of a framework bearing spherical air voids, which are arranged with facecentered cubic (fcc) close packing and are interconnected via the air channels, which exhibited iridescent colors immersed in water due to Bragg diffraction,,$^{3,5,46-48}$ indicating the existence of orderly periodic arrays of macropore. The reflective color can subtly be tuned by manipulating pore sizes of inverse opal with varying diameters of silica colloidal nanospheres. The typical in situ nanoindentation measurement of a photonic crystal membrane with $300 \mathrm{~nm}$ macropores shows an average Young's moduli of $55 \mathrm{MPa}$ under $300 \mu \mathrm{N}$ indentation force (Fig. S2 $\dagger$ ). Such a result was lower than that of pure polymer film without macropores (96 MPa). This is reasonable because the existence of macropores decreased the mechanical properties of polymer films. Fig. S3† shows that the photonic membrane is hydrophilic with a water contact angle of $19^{\circ}$.

The inverse opal photonic crystal membranes showed "cold" programming phenomenon after drying out of water at room temperature. The diffractive color gradually vanished as water evaporated, and a nearly colorless translucent appearance was shown. This phenomenon was not related to evaporation rate of water. The tilted cross-sectional scanning electron microscope (SEM) image of the water-dried membrane templated from $300 \mathrm{~nm} \mathrm{SiO}$ particles clearly shows collapsed and disordered nanostructure (Fig. 2a) with rough surface, which indicates the lost of orderly periodic structure and an order-to-disorder transition of elastic macropores after drying out of water. The corresponding atomic force microscopy (AFM, Fig. 2c and e) image further revealed the membrane was in an rough and uneven surface with root-mean-square linear profile roughness $\left(R_{\mathrm{q}}\right)$ of $49.4 \mathrm{~nm}$ (calculated according to the depth profile scanned across the line with $y=2.5 \mu \mathrm{m})$.

We suggested that large capillary pressure (additional pressure of curved liquid surface), produced by water evaporation from the template macropores due to its high surface tension $\left(72.75 \mathrm{mN} \mathrm{m}^{-1}\right.$ at $20^{\circ} \mathrm{C}$ ), was responsible for the formation of translucent copolymer membrane with collapsed nanostructures. It is known that capillary pressure was given by the Young-Laplace equation, $P=2 \gamma \cos \theta / R$, where $\gamma$ refers to the liquid/vapor surface tension, $\theta$ is the contact angle, and $R$ represents the radius of pores. ${ }^{42}$ The equation illustrates the higher the surface tension of solvent, the higher the capillary pressure. The smaller the radius of pores, the higher the capillary pressure. That is, the originally ordered macropores of 

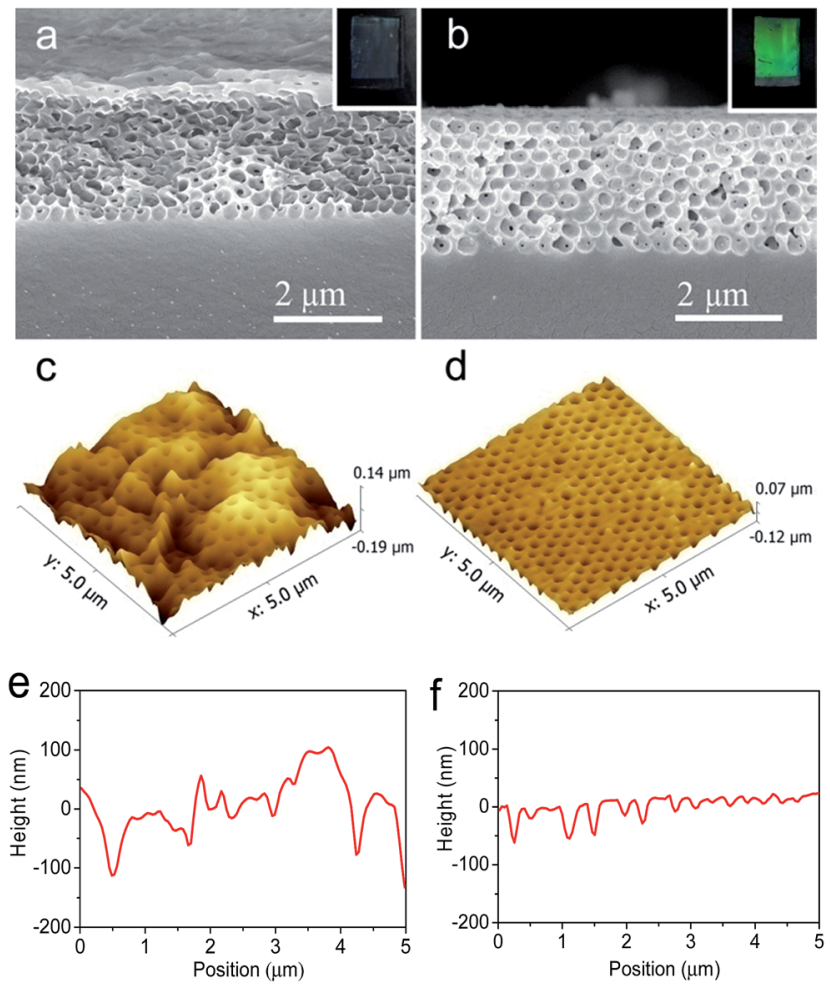

Fig. 2 Cross-sectional SEM images of the inverse opal photonic crystal membranes with $300 \mathrm{~nm}$ macropores after drying out of water (a) and heating recovery (b). The insets show photographs of the samples. AFM images and the corresponding depth profiles scanned across the line (with $y=2.5 \mu \mathrm{m}$ ) of the photonic crystal membranes after drying out of water ( $c$ and e) and heating recovery ( $d$ and f).

inverse opal photonic crystal membranes would be maintained, if solvent evaporation produced $P$ were smaller than elastic modulus (or Young's modulus) of the copolymer film. Small capillary pressure could be obtained when solvents with low surface tension and membranes with large pore sizes were used. To verify our hypothesis, two series of control experiments were conducted. First, ethanol and acetone liquids (with $\gamma$ of 22.39 and $23.7 \mathrm{mN} \mathrm{m}^{-1}$ for ethanol and acetone at $20^{\circ} \mathrm{C}$, respectively) were used as solvents, and the photonic crystal membranes were dried out of them. It is shown the membranes exhibited strong diffractive peaks as these solvents evaporated, which implied the appearance of highly ordered inverse opals photonic crystal nanostructures. Then, we fabricated inverse opals photonic crystal membranes templated from 240 and $350 \mathrm{~nm}$ silica microspheres (Fig. S4 and S5 $\dagger$ ), respectively. After drying out of water, the reflective peaks of membrane templated from $240 \mathrm{~nm}$ silica microspheres disappeared, while the membrane templated from $350 \mathrm{~nm}$ silica microspheres maintained highly reflective peaks and ordered nanostructure (Fig. S5 $\dagger$ ). Therefore, from the above discussion, it is demonstrated that the large capillary pressure caused by water evaporation from the template macropores was responsible for the "cold" programming. Accompanying with the collapse and deformation of periodic macropores, partially molecular chain segments of copolymer would be internally stressed..$^{49-51}$ The van der Waals forces between macromolecules in deformed macropores would constrain the motion of molecular chain segments, which stored internal energy (with lower entropic freedom) and rendered the collapsed structure remained, ${ }^{35,40}$ thereby forming highly stable temporary shape at room temperature.

The recovery of permanent inverse opal photonic crystal structure can be achieved by heating the deformed sample above $T_{\mathrm{g}}$ of copolymer. The deformed macroporous polymer membrane showed a immediate restoration of shining color once it was placed onto a hot plate at $80{ }^{\circ} \mathrm{C}$. Fig. $2 \mathrm{~b}$ shows the cross-sectional SEM image of recovered photonic crystal membrane with $300 \mathrm{~nm}$ macropores after heating. The high ordered arrays of macropores revealed the recovery of permanent shape with periodic inverse opal nanostructure. Moreover, AFM image (Fig. 2d) and the corresponding scanned depth profile (Fig. 3b) illustrate that the heat-recovered membrane has a much smoother surface (with $R_{\mathrm{q}}$ of $12.8 \mathrm{~nm}$ ) than the waterdried sample. These results indicate a disorder-order transition of copolymer membrane during heating.

In order to uncover this disorder-to-order transition, heatabsorption performance of photonic crystal membrane was measured by differential scanning calorimetry (DSC). To consistent with experiment environment, DSC measurement was carried out from room temperature to $250{ }^{\circ} \mathrm{C}$ at a heating rate of $10{ }^{\circ} \mathrm{C} \mathrm{min}^{-1}$. We can observe that inverse opal membrane had an endothermic peak at around $90{ }^{\circ} \mathrm{C}$ with a glass transition temperature of $80{ }^{\circ} \mathrm{C}$ (Fig. 3). The pure copolymer that had neither water treatment nor inverse opal structure showed a similar endothermic peak (Fig. S6†). It is indicated that the reconfigurable capability of inverse opal photonic crystal was attributed to the intrinsic property of PEG600DA-co-EO15TMPTA copolymer. When the photonic crystal membrane was heated to $80^{\circ} \mathrm{C}$, it transformed from the glass state to the rubber-elastic state. ${ }^{35,37}$ Heating could strengthen the motion of molecular and chain segments. Partially constrained molecular chain segments would be released to the originally internal stress-free state (stress-free configurations) with larger entropy under rubber-elastic state (entropy elasticity). ${ }^{39,40,52}$ As a result, the stored internal stress in deformed polymer chains of collapsed membranes was released, and thereby the disordered arrays recovered back to

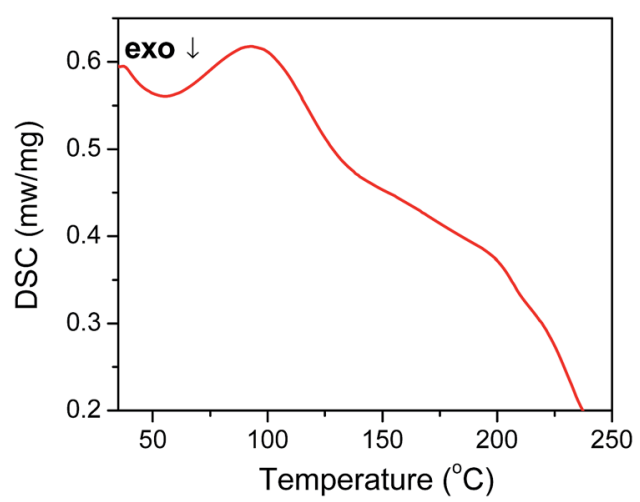

Fig. 3 Typical DSC plot of the photonic crystal membrane with $300 \mathrm{~nm}$ macropores. 
photonic crystal nanostructures with vivid diffractive color by heating over $T_{\mathrm{g}}$ of copolymer. This colored state was well maintained and highly stable, even when the membrane was cooled to room temperature.

To further investigate this disorder-to-order transition of photonic crystal membranes, the corresponding reflective spectra were measured using an optical spectrometer. The reflective spectra of the same sample with $300 \mathrm{~nm}$ macropores after water-drying (disordered temporary state) and heating (recovered permanent state) are shown in Fig. 4a. It is observed that water-dried sample showed no Bragg diffraction peaks due to the absence of ordered arrays, while the recovered membrane triggered by heating exhibited distinct diffraction peaks at $544 \mathrm{~nm}$ with well-defined Fabry-Perot fringes due to its highlyordered arrays. ${ }^{\mathbf{4 2 , 4 3 , 5 3}}$ According to the Bragg-Snell equation, the theoretical reflectance peak position is calculated to be $558 \mathrm{~nm}$. This result slightly larger than that of actually experimental measurement, because the interstitial of template was not infiltrated completely. Additionally, the reflective intensities of photonic crystal membranes dried out of ethanol and acetone are slightly lower than that of the heating-recovered one as shown in Fig. 4a. The smaller reflective intensity indicates ethanol- and acetone-triggered recovery of photonic macropores is not as complete as the heating-induced recovery, as the
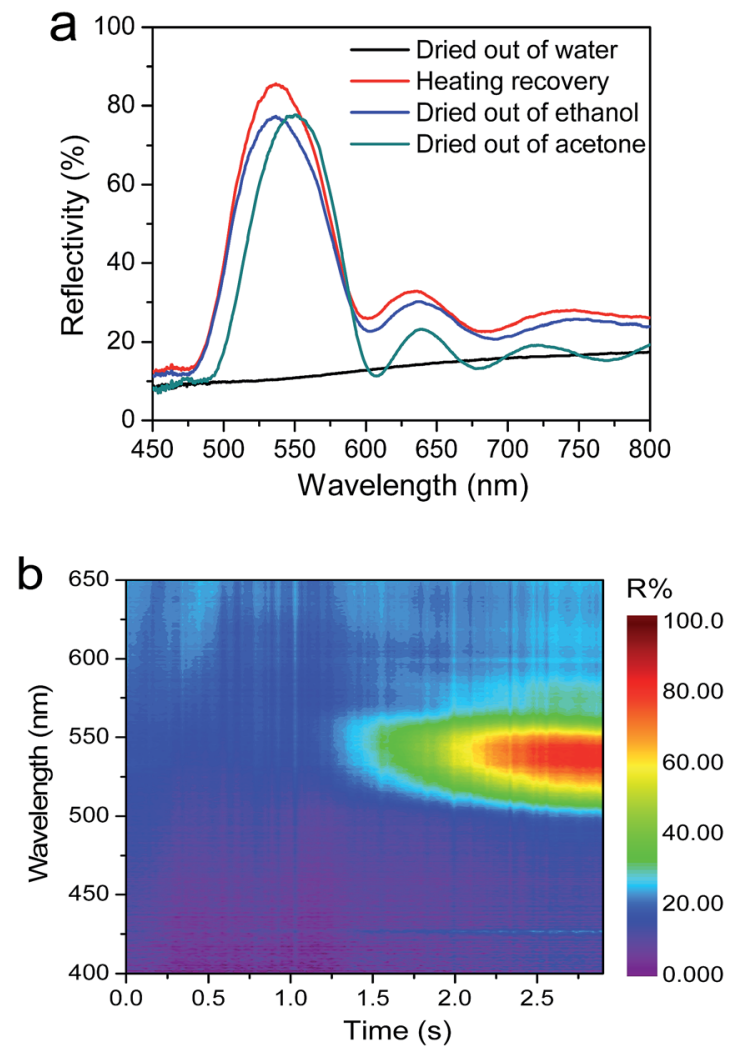

Fig. 4 Normal-incidence optical reflection spectra obtained from the photonic crystal membrane with $300 \mathrm{~nm}$ macropores (a) after drying out of water, acetone and ethanol, and heating recovery. (b) 2D plot of time-resolved optical reflection spectra during thermally induced recovering of the corresponding membrane. reflective intensity is sensitive to its crystalline thickness. ${ }^{41}$ As for the difference in diffraction peak position, we ascribe this to high swelling ratio of photonic crystal membrane in acetone (Fig. S7†). Similar diffraction spectra were also observed in those samples exposed to acetone and ethanol liquids. Further insight into recovering process was revealed by dynamic reflective spectra. Fig. $4 \mathrm{~b}$ exhibits the corresponding timeresolved normal-incidence optical reflection spectra of collapsed membrane, which was placed on a hot plate of $80{ }^{\circ} \mathrm{C}$ with photonic crystal layer facing down. A rapid rise in the amplitude of characteristic reflection band was shown and a plateau was reached after around $2 \mathrm{~s}$ (Fig. $\mathrm{S} 8 \dagger$ ). This results suggests a fast recovery of inverse opal nanostructures, which is important for developing real-time response photonic crystal devices. Because of high stability of both disordered and ordered nanostructures, the current photonic crystal membranes exhibited high stable optical reflection characteristics, thereby implying the presence of optical bistability.

Along with the fast recovery, reversibility and durability are also key parameters for practical applications of these heatinduced reconfigurable membranes. Fig. 5 shows the reflective spectra of the membrane cyclically exposed to heat-recovery and then dried out of water for ten times. The collapsed and recovered states exhibit almost the same color appearances and reflective intensities, revealing the high reversibility and durability.
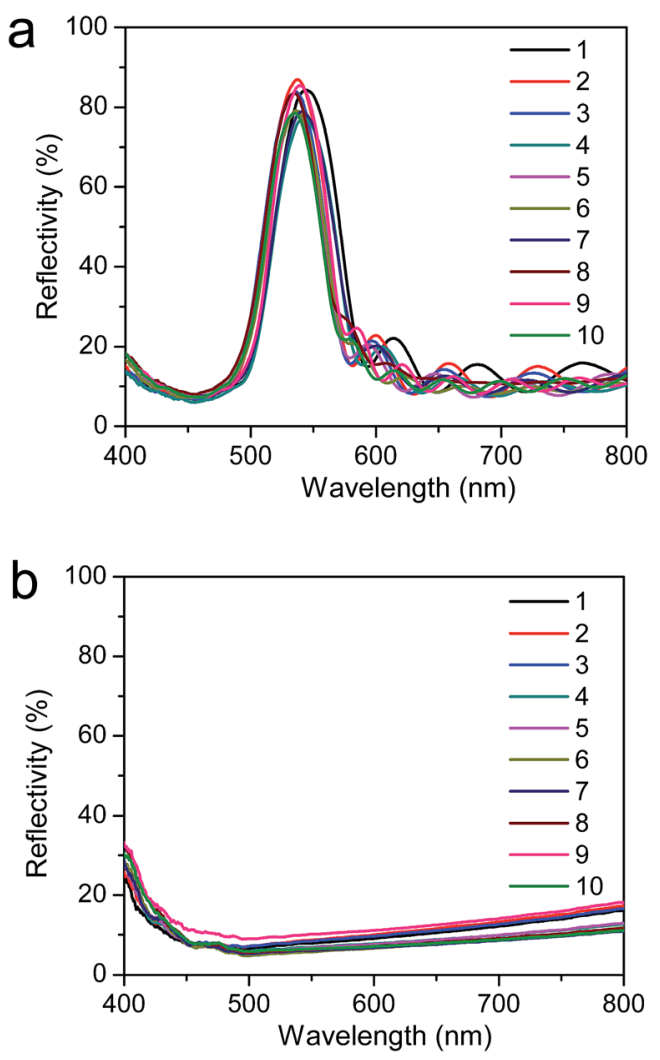

Fig. 5 Normal-incidence optical reflectance spectra obtained from the photonic crystal membrane with $300 \mathrm{~nm}$ macropores cyclically (a) heating recovery and (b) dried out of water. 
To demonstrate potential application for photoswitches, we measured the transmittance of photonic crystal membranes during "cold" programming and heat recovery processes (Fig. S9 $\dagger$ ). Accompanying with the collapse of ordered elastic arrays, water evaporation-induced large capillary pressure also resulted in a sharply change of transmittance (or photonic bandgaps), as $T \%$ is $1-A \%-R \%-S \%$ ( $T, A, R$ and $S$ present transmittance, absorbance, reflectance and scattering, respectively) ${ }^{54-56}$ This is critical for a photoswitch because a large difference of transmittances means a higher contrast and on/ off ratio. ${ }^{54-56}$ It can be observed that an average transmittance of $70 \%$ was reached at $544 \mathrm{~nm}$ in collapsed sample with disordered arrays (Fig. 6). The optical loss of around $30 \%$ was attributed to absorption and scattering of membrane. When inverse opal structure with ordered arrays were recovered by heating, the reflective peaks of photonic crystal membrane appeared, thereby leading to an average transmittance of as low as $5 \%$ at $544 \mathrm{~nm}$. No apparent degradation in their optical performances was shown during cyclically measurements for ten times. This large difference of transmittances between the permanent (ordered) and the temporary (disordered) states implied that the reversible transition between "off" and "on" states of a photonic crystal photoswitch could be easily achieved. Moreover, for most of conventional photonic crystal photoswitches, "on-off" transition is usually realized through
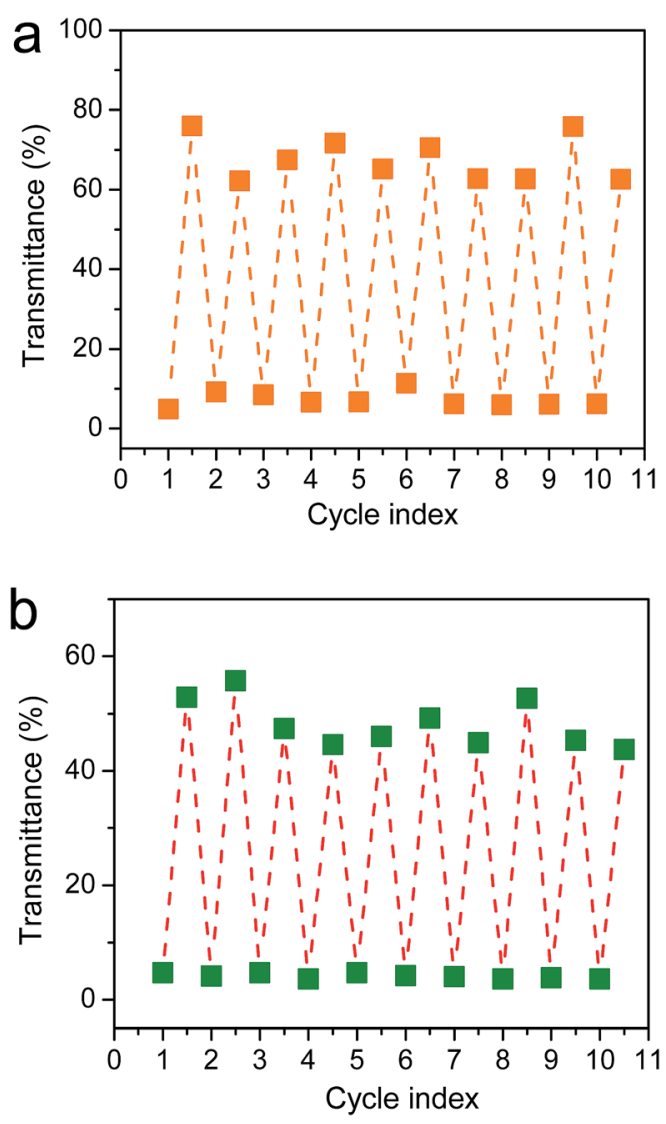

Fig. 6 Optical transmittance amplitudes obtained from the photonic crystal films with $300 \mathrm{~nm}$ ((a) at $544 \mathrm{~nm}$ ) and $240 \mathrm{~nm}$ ((b) at $445 \mathrm{~nm}$ ) macropores cyclically deformed and recovered for ten times. the shift of photonic bandgap or the migration of defect mode. ${ }^{57-59}$ However, optical switching effect and/or contrast were usually limited by the slope of photonic bandgap edges due to small shift of reflective peaks $(\Delta \lambda)$. By contrast, the present photonic crystal membrane exhibited a large $\Delta \lambda$ (it equals to $\lambda_{\max }$ of the photonic crystal) as no reflective peaks existed in the deformed state, which provides a unique alternative in developing photonic crystal photoswitches with higher contrast and optical switching effect. Similarly, the effective management of $445 \mathrm{~nm}$ light signal was also achieved in inverse opal membranes with $240 \mathrm{~nm}$ macropores (Fig. 6b and $\mathrm{S} 10 \dagger)$.

\section{Experimental section}

\section{Materials}

Tetraethylsilicate (99.5\%, AR) and ammonia solution (25\%, AR) were purchased from Tianjin Damao Chemical Reagents. Ethanol (99.7\%, AR) was purchased from Sinopharm Chemical Reagents. Polyethylene glycol (600) diacrylate (PEG600DA, Agisyn2835) and ethoxylated (15) trimethylolpropane triacrylate (EO15TMPTA) were provided by DSM Chemical Co. Ltd. All reagents were used as received without further purification.

\section{Preparation of reconfigurable photonic crystals}

Monodispersed silica microspheres were synthesized according to a modified Stöber method, by adding $\mathrm{NaCl}$ as electrolyte to improve the surface charge of silica particles. The resulting silica microspheres were purified in ethanol by centrifugation and redispersion cycles at least for three times. Then, the purified silica microspheres were self-assembled into 3D photonic crystals on a hydrophilic processed microslide by vertical assembly method under constant temperature and humidity environment. The self-assembled silica templates were infiltrated with a small amount of viscous oligomer mixtures, consisting of PEG600DA and EO15TMPTA (the mass ratio was 1 : 4, and light initiator Darocur 2-hydroxy-2-methyl-1phenyl-1-propanone 1173 with mass fraction of $1 \%$ was added) at $60{ }^{\circ} \mathrm{C}$. The microslide with silica photonic crystal template was covered by another microslide, separated by $1 \mathrm{~mm}$ thick double-sided adhesive tapes. The air interstitials in this sandwich cell were filled up with monomer mixture, which was then irradiated using a combined $254 \mathrm{~nm}$ and $365 \mathrm{~nm}$ UV light source $(12 \mathrm{~W})$ for $1 \mathrm{~min}$ to initiate polymerization. In order to completely removing silica template, the film was finally soaked in a 4 vol\% $\mathrm{HF}$ aqueous solution for $6 \mathrm{~h}$ and rinsed with deionized water, which got inverse opal membrane in $1 \mathrm{~mm}$ thickness.

\section{Shape memory programming and recovery of photonic crystal}

The "cold" programming was performed by applying a layer of water and then dried out of it. To recover the permanent ordered inverse opal photonic crystal, the deformed membrane was placed onto a hot plate (at $80{ }^{\circ} \mathrm{C}$ ) for $8 \mathrm{~s}$ with photonic crystal layer facing down. 


\section{Sample characterization}

SEM image was carried out on an FEI Nova NanoSEM 450 with 10 $\mathrm{kV}$ accelerating voltage. A thin gold layer was sputtered onto the samples prior to imaging for $30 \mathrm{~s}$. Atomic force microscopy (AFM) image was performed using a Park Systems XE-70 AFM. In situ nanoindentation tests were performed with an MTS nanoindenter XP system using a spherical sapphire indenter. Differential scanning calorimetry measurements were measured from room temperature to $250{ }^{\circ} \mathrm{C}$ at a heating rate of $10{ }^{\circ} \mathrm{C} \mathrm{min} \mathrm{m}^{-1}$ using a Netzsch DSC 204 and an empty pan as reference. The weight of specimens was $10 \mathrm{mg}$. Normal-incidence optical reflection and transmission spectra were obtained using a fiber optic spectrometer PG2000-pro with a tungsten halogen light source.

\section{Conclusions}

In conclusion, we have presented a type of reconfigurable photonic crystal membranes with optically bistable states, which was enabled by capillary pressure-induced "cold" programming and heat-caused recoverable shape memory polymers. The large capillary pressure and heat-recovery resulted in reversible transition of photonic crystal between a permanent ordered state and a disordered temporary state during the cyclical programming and recovery, which created tremendous changes in appearance and diffractive performances. This reconfigurable photonic crystal phenomenon could be used as a unique optical technique to probe shape memory effect at nanoscale. Simultaneously, a large and stable shift of photonic bandgaps in this optically bistable photonic crystal provides an alternative to developing photonic crystal photoswitches with higher contrast, as well as rewritable photonic devices and displays etc. Also, heat-induced recovery or opening of macropores may find applications in smart interface and drug delivery by manipulating $T_{\mathrm{g}}$ of system.

\section{Acknowledgements}

The work was financially supported by National Natural Science Foundation of China (21506023), Key Program of National Natural Science Foundation of China (21536002), Doctoral Research Foundation of Liaoning Province (201501170), the Fund for innovative research groups of the National Natural Science Fund Committee of Science (21421005) and Fundamental Research Funds for the Central Universities (DUT15RC(3)015, DUT15ZD224, DUT2016TB12).

\section{Notes and references}

1 S. Ogawa, M. Imada, S. Yoshimoto, M. Okano and S. Noda, Science, 2004, 305, 227.

2 O. D. Velev, A. M. Lenhoff and E. W. Kaler, Science, 2000, 287, 2240.

3 L. D. Bonifacio, B. V. Lotsch, D. P. Puzzo, F. Scotognella and G. A. Ozin, Adv. Mater., 2009, 21, 1641.
4 Y. Cho, S. Y. Lee, L. Ellerthorpe, G. Feng, G. Lin, G. Wu, J. Yin and S. Yang, Adv. Funct. Mater., 2015, 25, 6041.

5 K. Ueno, A. Inaba, Y. Sano, M. Kondoh and M. Watanabe, Chem. Commun., 2009, 3603.

6 J. Ge and Y. Yin, Angew. Chem., Int. Ed., 2011, 50, 1492.

7 Y. Yue and J. P. Gong, J. Photochem. Photobiol., C, 2015, 23, 45.

8 C. Fenzl, T. Hirsch and O. S. Wolfbeis, Angew. Chem., Int. Ed., 2014, 53, 3318.

9 X. Hu, Q. An, G. Li, S. Tao and J. Liu, Angew. Chem., Int. Ed., 2006, 45, 8145.

10 A. Pal, V. Malik, L. He, B. H. Erne, Y. Yin, W. K. Kegel and A. V. Petukhov, Angew. Chem., Int. Ed., 2015, 54, 1803.

11 M. Qin, Y. Huang, Y. Li, M. Su, B. Chen, H. Sun, P. Yong, C. Ye, F. Li and Y. Song, Angew. Chem., Int. Ed., 2016, 55, 6911.

12 H. He, S. Averick, P. Mandal, H. Ding, S. Li, J. Gelb, N. Kotwal, A. Merkle, S. Litster and K. Matyjaszewski, Adv. Sci., 2015, 2, 1500069.

13 D. Yang, Y. Qin, S. Ye and J. Ge, Adv. Funct. Mater., 2014, 24, 817.

14 D. Yang, S. Ye and J. Ge, Adv. Funct. Mater., 2014, 24, 3197. 15 S. Ye, Q. Fu and J. Ge, Adv. Funct. Mater., 2014, 24, 6430.

16 A. C. Arsenault, D. P. Puzzo, I. Manners and G. A. Ozin, Nat. Photonics, 2007, 1, 468.

17 H. Li, J. Wang, H. Lin, L. Xu, W. Xu, R. Wang, Y. Song and D. Zhu, Adv. Mater., 2010, 22, 1237.

18 J. Hou, H. Zhang, Q. Yang, M. Li, Y. Song and L. Jiang, Angew. Chem., Int. Ed., 2014, 53, 5791.

19 Y. Yue, T. Kurokawa, M. A. Haque, T. Nakajima, T. Nonoyama, X. Li, I. Kajiwara and J. P. Gong, Nat. Commun., 2014, 5, 4659.

20 J. Hou, H. Zhang, Q. Yang, M. Li, L. Jiang and Y. Song, Small, 2015, 11, 2738.

21 H. Jiang, Y. Zhu, C. Chen, J. Shen, H. Bao, L. Peng, X. Yang and C. Li, New J. Chem., 2012, 36, 1051.

22 Y. Zhang, Q. Fu and J. Ge, Nat. Commun., 2015, 6, 7510.

23 I. Pavlichenko, A. T. Exner, M. Guehl, P. Lugli, G. Scarpa and B. V. Lotsch, J. Phys. Chem. C, 2012, 116, 298.

24 S. H. Kim, J. G. Park, T. M. Choi, V. N. Manoharan and D. A. Weitz, Nat. Commun., 2014, 5, 3068.

25 J. Y. Kim, H. Kim, B. H. Kim, T. Chang, J. Lim, H. M. Jin, J. H. Mun, Y. J. Choi, K. Chung, J. Shin, S. Fan and S. O. Kim, Nat. Commun., 2016, 7, 12911.

26 M. G. Han, C. J. Heo, H. Shim, C. G. Shin, S. J. Lim, J. W. Kim, Y. W. Jin and S. Lee, Adv. Opt. Mater., 2014, 2, 535.

27 C. Zhu, W. Xu, L. Chen, W. Zhang, H. Xu and Z. Z. Gu, Adv. Funct. Mater., 2011, 21, 2043.

28 M. G. Han, C. G. Shin, S. J. Jeon, H. Shim, C. J. Heo, H. Jin, J. W. Kim and S. Lee, Adv. Mater., 2012, 24, 6438.

29 L. He, M. Wang, J. GE and Y. Yin, Acc. Chem. Res., 2012, 45, 1431.

30 E. P. Chan, J. J. Walish, A. M. Urbas and E. L. Thomas, $A d v$. Mater., 2013, 25, 3934.

31 W. Wang, N. Xie, L. He and Y. Yin, Nat. Commun., 2014, 5, 5459.

32 T. Ding, Q. Zhao, S. K. Smoukov and J. J. Baumberg, Adv. Opt. Mater., 2014, 2, 1098. 
33 (a) Y. A. Vlasov, X. Z. Bo, J. C. Sturm and D. J. Norris, Nature, 2001, 414, 289; (b) H. F. Zhang and S. B. Liu, AIP Adv., 2016, 6, 085116; (c) H. F. Zhang and S. B. Liu, Opt. Quantum Electron., 2016, 48, 508; (d) H. F. Zhang, S. B. Liu and X. K. Kong, Solid State Commun., 2012, 152, 2113.

34 Y. Fang, Y. Ni, S. Y. Leo, B. Wang, V. Basile, C. Taylor and P. Jiang, ACS Appl. Mater. Interfaces, 2015, 7, 23650.

35 T. Xie, Nature, 2010, 464, 267.

36 A. Rose, Z. Zhu, C. F. Madigan, T. M. Swager and V. Bulovic, Nature, 2005, 434, 876.

37 X. Feng, G. Zhang, B. Xu, H. Jiang, Q. Bai and H. Li, RSC Adv., 2015, 5, 70000.

38 I. Bellin, S. Kelch, R. Langer and A. Lendlein, Proc. Natl. Acad. Sci. U. S. A., 2006, 103, 18043.

39 A. Espinha, M. C. Serrano, Á. Blanco and C. López, Adv. Opt. Mater., 2014, 2, 6.

40 Z. Wang, C. Hansen, Q. Ge, S. H. Maruf, D. U. Ahn, H. J. Qi and Y. Ding, Adv. Mater., 2011, 23, 3669.

41 Y. Fang, S.-Y. Leo, Y. Ni, L. Yu, P. Qi, B. Wang, V. Basile, C. Taylor and P. Jiang, Adv. Opt. Mater., 2015, 3, 1509.

42 Y. Fang, Y. Ni, B. Choi, S. Y. Leo, J. Gao, B. Ge, C. Taylor, V. Basile and P. Jiang, Adv. Mater., 2015, 27, 3696.

43 Y. Fang, Y. Ni, S. Y. Leo, C. Taylor, V. Basile and P. Jiang, Nat. Commun., 2015, 6, 7416.

44 Y. Ohtsuka, T. Seki and Y. Takeoka, Angew. Chem., Int. Ed., 2015, 54, 15368.

45 J. Li, Y. Wu, J. Fu, Y. Cong, J. Peng and Y. Han, Chem. Phys. Lett., 2004, 390, 285.
46 G. Guidetti, S. Atifi, S. Vignolini and W. Y. Hamad, Adv. Mater., 2016, 28, 10042.

47 L. Shang, F. Shangguan, Y. Cheng, J. Lu, Z. Xie, Y. Zhao and Z. Gu, Nanoscale, 2013, 5, 9553.

48 F. Wang, X. Zhang, Y. Lin, L. Wang and J. Zhu, ACS Appl. Mater. Interfaces, 2016, 8, 5009.

49 D. Habault, H. Zhang and Y. Zhao, Chem. Soc. Rev., 2013, 42, 7244.

50 Y. Zhang, Q. Wang, C. Wang and T. Wang, J. Mater. Chem., 2011, 21, 9073.

51 R. Luo, J. Wu, N.-D. Dinh and C. H. Chen, Adv. Funct. Mater., 2015, 25, 7272.

52 J. Y. Ou, E. Plum, L. Jiang and N. I. Zheludev, Nano Lett., 2011, 11, 2142.

53 M. Kuang, J. Wang, B. Bao, F. Li, L. Wang, L. Jiang and Y. Song, Adv. Opt. Mater., 2014, 2, 34.

54 B. Rosner, M. Milek, A. Witt, B. Gobaut, P. Torelli, R. H. Fink and M. M. Khusniyarov, Angew. Chem., Int. Ed., 2015, 54, 12976.

55 G. Wang, D. Yuan, T. Yuan, J. Dong, N. Feng and G. Han, J. Polym. Sci., Part A: Polym. Chem., 2015, 53, 2768.

56 M. Irie, T. Fukaminato, K. Matsuda and S. Kobatake, Chem. Rev., 2014, 114, 12174.

57 C. C. Tartan, P. S. Salter, M. J. Booth, S. M. Morris and S. J. Elston, J. Appl. Phys., 2016, 119, 183106.

58 J. Guo, Y. Liu, Z. Wang, M. Luo, W. Huang, T. Han and X. Liu, J. Opt., 2016, 18, 055706.

59 M. Shirdel and M. A. Mansouri-Birjandi, Optik, 2016, 127, 3955. 